\title{
Lax Representations for Separable Systems from Benenti Class
}

\author{
Maciej BEASZAK ${ }^{\dagger}$ and Ziemowit DOMAŃSKI \\ $\dagger$ Faculty of Physics, Division of Mathematical Physics, A. Mickiewicz University, \\ Uniwersytetu Poznańskiego 2, 61-614 Poznań, Poland \\ E-mail: blaszakm@amu.edu.pl \\ ¥ Institute of Mathematics, Poznań University of Technology, \\ Piotrowo 3A, 60-965 Poznań, Poland \\ E-mail: ziemowit.domanski@put.poznan.pl
}

Received December 13, 2018, in final form June 07, 2019; Published online June 18, 2019 https://doi.org/10.3842/SIGMA.2019.045

\begin{abstract}
In this paper we construct Lax pairs for Stäckel systems with separation curves from so-called Benenti class. For each system of considered family we present an infinite family of Lax representations, parameterized by smooth functions of spectral parameter.
\end{abstract}

Key words: Lax representation; Stäckel system; Benenti system; Hamiltonian mechanics

2010 Mathematics Subject Classification: 70H06; 37J35

\section{Introduction}

Classical Stäckel systems belong to important class of integrable and separable Hamiltonian ODE's. The constants of motion of these systems are quadratic in momenta and describe many physical systems of classical mechanics. The Stäckel systems are defined by separation relations, i.e., relations involving canonical variables $\left(\lambda_{i}, \mu_{i}\right)_{i=1, \ldots, n}$ in which the Hamilton-Jacobi equations separate to a system of decoupled ordinary differential equations. The separation relations of classical Stäckel system are represented by $n$ algebraic equations of the form

$$
\sigma_{i}\left(\lambda_{i}\right)+\sum_{k=1}^{n} H_{k} S_{i k}\left(\lambda_{i}\right)=\frac{1}{2} f_{i}\left(\lambda_{i}\right) \mu_{i}^{2}, \quad i=1,2, \ldots, n,
$$

where $n$ is the number of degrees of freedom of the system, i.e., $2 n$ is the dimension of the corresponding phase space on which the system is defined, $H_{1}, H_{2}, \ldots, H_{n}$ are $n$ Hamiltonians, $f, \sigma$ and $S$ are arbitrary smooth functions. Solving the system (1.1), under assumption that $\operatorname{det} S \neq 0$, with respect to all $H_{i}$ we get $n$ Hamiltonians expressed in variables $\left(\lambda_{i}, \mu_{i}\right)_{i=1, \ldots, n}$, which from construction will be in involution, i.e., their Poisson brackets vanish $\left\{H_{i}, H_{j}\right\}=0$, and which Hamilton-Jacobi equations separate. In other words the system (1.1) describes a Liouville-integrable and separable Hamiltonian system.

The special, but particularly important class of Stäckel systems is the Benenti class $[1,2,3]$. This class is described by the following separation relations

$$
\sigma_{i}\left(\lambda_{i}\right)+H_{1} \lambda_{i}^{n-1}+H_{2} \lambda_{i}^{n-2}+\cdots+H_{n}=\frac{1}{2} f_{i}\left(\lambda_{i}\right) \mu_{i}^{2}, \quad i=1,2, \ldots, n .
$$

There is an extended literature on systems from class (1.2), however less can be found on their Lax representation. A Lax representation of a Liouville integrable Hamiltonian system is 
a set of matrices $L, U_{k}(k=1,2, \ldots, n)$ which satisfy the system of Lax equations

$$
\frac{\mathrm{d} L}{\mathrm{~d} t_{k}}=\left[U_{k}, L\right], \quad k=1,2, \ldots, n,
$$

under the assumption that the time evolution with respect to $t_{k}$ is governed by the Hamiltonian vector field $X_{H_{k}}$.

In the paper we find an infinite family of Lax representations for an arbitrary Stäckel system from the Benenti class generated by the following canonical form of separation curves

$$
\sigma(\lambda)+\sum_{k=1}^{n} H_{k} \lambda^{n-k}=\frac{1}{2} f(\lambda) \mu^{2},
$$

where the spectral parameter $\lambda$ is real and the functions $f(\lambda)$ and $\sigma(\lambda)$ are smooth and real valued. Separation relations (1.2) are reconstructed by $n$ copies of $(1.4)$ with $\left(\lambda_{i}, \mu_{i}\right)_{i=1, \ldots, n}$. In literature, the reader can find Lax representation for systems related to various subcases of separation curves from the family (1.4). First constructions of Lax representation for separable systems with separation curves of particular hyperelliptic form was constructed by Mumford [10]. For $\sigma(\lambda)$ in polynomial form and $f(\lambda)=\lambda^{2 r}, r \in \mathbb{N}$, Lax equations were constructed explicitly in [14] and analyzed in particular coordinate frames. In [8], using different technique, Lax representation was constructed for the subclass of separation curves (1.4) with $f(\lambda)$ being polynomials of order $n+1, n$ and $n-1$, respectively, with distinct roots. Yet another subcases of family (1.4) together with the construction of Lax representations by various techniques, the reader can find in $[11,13]$.

Here we present the explicit form of infinite family of admissible Lax representations, for systems generated by (1.4) with $\sigma(\lambda)$ and $f(\lambda)$ being arbitrary smooth real valued functions, in separation coordinates and in so called Viète coordinates.

The paper is organized as follows. In Section 2 we present basic facts about Benenti systems. In Section 3 we present in explicit form the infinite family of Lax matrices $L(\lambda)$ and Lax equations (1.3) in separation coordinates and state that the characteristic equation of each Lax matrix corresponds to the separation curve (1.4) of Benenti system. All results of this section we gather in Theorem 3.2, which we prove in Section 4. Section 5 contains the particular Lax representations in Viète coordinates in which many formulas simplify. Section 6 contains several examples illustrating the theory. We end the article with some comments concerning the main result and its further application.

\section{Preliminaries}

Let us consider separable systems generated by separation curves for canonical coordinates in the form (1.4). The Poisson bracket in canonical coordinates $\left(\lambda_{i}, \mu_{i}\right)_{i=1, \ldots, n}$ takes the canonical form

$$
\{f, g\}=\sum_{i=1}^{n}\left(\frac{\partial f}{\partial \lambda_{i}} \frac{\partial g}{\partial \mu_{i}}-\frac{\partial f}{\partial \mu_{i}} \frac{\partial g}{\partial \lambda_{i}}\right) .
$$

In particular

$$
\left\{\lambda_{i}, \mu_{j}\right\}=\delta_{i j}, \quad\left\{\lambda_{i}, \lambda_{j}\right\}=\left\{\mu_{i}, \mu_{j}\right\}=0 .
$$

By taking $n$ copies of (1.4) with $(\lambda, \mu)=\left(\lambda_{i}, \mu_{i}\right), i=1, \ldots, n$, we get a system of $n$ linear equations for $H_{k}$ :

$$
H_{1} \lambda_{1}^{n-1}+H_{2} \lambda_{1}^{n-2}+\cdots+H_{n}=F\left(\lambda_{1}, \mu_{1}\right),
$$




$$
\begin{aligned}
& H_{1} \lambda_{2}^{n-1}+H_{2} \lambda_{2}^{n-2}+\cdots+H_{n}=F\left(\lambda_{2}, \mu_{2}\right), \\
& H_{1} \lambda_{n}^{n-1}+H_{2} \lambda_{n}^{n-2}+\cdots+H_{n}=F\left(\lambda_{n}, \mu_{n}\right),
\end{aligned}
$$

where $F(x, y)=\frac{1}{2} f(x) y^{2}-\sigma(x)$. Its solution gives us $n$ real valued Hamiltonians

$$
H_{k}(\lambda, \mu)=E_{k}(\lambda, \mu)+V_{k}(\lambda)=-\sum_{i=1}^{n} \frac{\partial \rho_{k}}{\partial \lambda_{i}} \frac{F\left(\lambda_{i}, \mu_{i}\right)}{\Delta_{i}},
$$

and related evolution equations

$$
\frac{\mathrm{d} \lambda_{i}}{\mathrm{~d} t_{k}}=\left\{\lambda_{i}, H_{k}\right\}, \quad \frac{\mathrm{d} \mu_{i}}{\mathrm{~d} t_{k}}=\left\{\mu_{i}, H_{k}\right\}, \quad i, k=1, \ldots, n,
$$

where

$$
\rho_{k}=(-1)^{k} s_{k}, \quad \Delta_{i}=\prod_{k \neq i}\left(\lambda_{i}-\lambda_{k}\right)
$$

and $s_{k}$ are elementary symmetric polynomials. From the linearity of (1.2), geodesic parts $E_{k}$ are defined by

$$
\sum_{j=1}^{n} E_{j} \lambda_{i}^{n-j}=\frac{1}{2} f\left(\lambda_{i}\right) \mu_{i}^{2}, \quad i=1, \ldots, n,
$$

and in canonical coordinates $\left(\lambda_{i}, \mu_{i}\right)_{i=1, \ldots, n}$ take the form [4]

$$
E_{j}(\lambda, \mu)=-\frac{1}{2} \sum_{i=1}^{n} \frac{\partial \rho_{j}}{\partial \lambda_{i}} \frac{f\left(\lambda_{i}\right) \mu_{i}^{2}}{\Delta_{i}}=\frac{1}{2} \sum_{i=1}^{n}\left(K_{j} G\right)^{i i} \mu_{i}^{2},
$$

where $G$ is contravariant metric tensor defined by $E_{1}$ and $K_{j}$ are Killing tensors of $G$ :

$$
G^{r s}=\frac{f\left(\lambda_{r}\right)}{\Delta_{r}} \delta^{r s}, \quad\left(K_{j}\right)_{s}^{r}=-\frac{\partial \rho_{j}}{\partial \lambda_{r}} \delta_{s}^{r} .
$$

Potentials $V_{k}$, defined by

$$
\sigma\left(\lambda_{i}\right)+\sum_{j=1}^{n} V_{j} \lambda_{i}^{n-j}=0, \quad i=1, \ldots, n
$$

take the form

$$
V_{k}(\lambda)=\sum_{i=1}^{n} \frac{\partial \rho_{k}}{\partial \lambda_{i}} \frac{\sigma\left(\lambda_{i}\right)}{\Delta_{i}}
$$

In particular, for $\sigma(\lambda)=\lambda^{\gamma}, \gamma \in \mathbb{Z}$, the corresponding potentials $V_{k}^{(\gamma)}$, called basic potentials, are constructed by the formula [7]

$$
V^{(\gamma)}=R^{\gamma} V^{(0)}, \quad V^{(\gamma)}=\left(V_{1}^{(\gamma)}, \ldots, V_{n}^{(\gamma)}\right)^{\mathrm{T}},
$$

where

$$
R=\left(\begin{array}{cccc}
-\rho_{1} & 1 & 0 & 0 \\
\vdots & 0 & \ddots & 0 \\
\vdots & 0 & 0 & 1 \\
-\rho_{n} & 0 & 0 & 0
\end{array}\right), \quad R^{-1}=\left(\begin{array}{cccc}
0 & 0 & 0 & -\frac{1}{\rho_{n}} \\
1 & 0 & 0 & \vdots \\
0 & \ddots & 0 & \vdots \\
0 & 0 & 1 & -\frac{\rho_{n-1}}{\rho_{n}}
\end{array}\right)
$$


and $V^{(0)}=(0, \ldots, 0,-1)^{\mathrm{T}}$. Notice that for $\gamma=0, \ldots, n-1$

$$
V_{k}^{(\gamma)}=-\delta_{k, n-\gamma}
$$

that is

$$
V^{(0)}=(0, \ldots, 0,-1)^{\mathrm{T}}, \quad \ldots, \quad V^{(n-1)}=(-1,0, \ldots, 0)^{\mathrm{T}} .
$$

The first nontrivial positive potential is

$$
V^{(n)}=\left(\rho_{1}, \ldots, \rho_{n}\right)^{\mathrm{T}}
$$

and the negative one

$$
V^{(-1)}=\left(\frac{1}{\rho_{n}}, \ldots, \frac{\rho_{n-1}}{\rho_{n}}\right)^{\mathrm{T}} .
$$

\section{Lax representation in separation coordinates}

In what follows we will assume that the functions $f(\lambda)$ and $\sigma(\lambda)$ appearing in the separation curve (1.4) are smooth, real valued and defined on an open subset $\mathcal{U} \subset \mathbb{R}$ such that for any phase space point $\left(\lambda_{1}, \ldots, \lambda_{n}, \mu_{1}, \ldots, \mu_{n}\right)$ each $\lambda_{i} \in \mathcal{U}, i=1,2, \ldots, n$. Moreover, we will require that $f(\lambda) \neq 0$ for every $\lambda \in \mathcal{U}$. In order to describe the Lax representation let us investigate the division of a smooth function on $\mathcal{U}$ by a polynomial.

Lemma 3.1. Let $b(\lambda)$ be a smooth function on $\mathcal{U}$ and

$$
a(\lambda)=\left(\lambda-\lambda_{1}\right)\left(\lambda-\lambda_{2}\right) \cdots\left(\lambda-\lambda_{n}\right)
$$

be a polynomial of order $n$ whose roots $\lambda_{1}, \lambda_{2}, \ldots, \lambda_{n} \in \mathcal{U}$, then the fraction $\frac{b(\lambda)}{a(\lambda)}$ can be uniquely written as

$$
\frac{b(\lambda)}{a(\lambda)}=h(\lambda)+\frac{r(\lambda)}{a(\lambda)},
$$

where $h(\lambda)$ is a smooth function on $\mathcal{U}$ and $r(\lambda)$ is a polynomial of order less than $n$.

Proof. We calculate that

$$
\begin{aligned}
\frac{b(\lambda)}{a(\lambda)} & =\frac{h_{1}(\lambda)}{\left(\lambda-\lambda_{2}\right)\left(\lambda-\lambda_{3}\right) \cdots\left(\lambda-\lambda_{n}\right)}+\frac{h_{0}\left(\lambda_{1}\right)}{a(\lambda)} \\
& =\frac{h_{2}(\lambda)}{\left(\lambda-\lambda_{3}\right)\left(\lambda-\lambda_{4}\right) \cdots\left(\lambda-\lambda_{n}\right)}+\frac{h_{0}\left(\lambda_{1}\right)+h_{1}\left(\lambda_{2}\right)\left(\lambda-\lambda_{1}\right)}{a(\lambda)}=\cdots \\
& =h_{n}(\lambda)+\frac{h_{0}\left(\lambda_{1}\right)+h_{1}\left(\lambda_{2}\right)\left(\lambda-\lambda_{1}\right)+\cdots+h_{n-1}\left(\lambda_{n}\right)\left(\lambda-\lambda_{1}\right) \cdots\left(\lambda-\lambda_{n-1}\right)}{a(\lambda)},
\end{aligned}
$$

where

$$
h_{0}(\lambda)=b(\lambda), \quad h_{i}(\lambda)=\frac{h_{i-1}(\lambda)-h_{i-1}\left(\lambda_{i}\right)}{\lambda-\lambda_{i}}, \quad i=1,2, \ldots, n .
$$

From the Taylor theorem we can see that each function $h_{i}(\lambda)$ is smooth on $\mathcal{U}$. In particular, we have that

$$
\lim _{\lambda \rightarrow \lambda_{i}} \frac{\mathrm{d}^{k}}{\mathrm{~d} \lambda^{k}} h_{i}(\lambda)=\frac{1}{k+1} \frac{\mathrm{d}^{k+1}}{\mathrm{~d} \lambda^{k+1}} h_{i-1}\left(\lambda_{i}\right) .
$$


Putting $h(\lambda)=h_{n}(\lambda)$ and $r(\lambda)=h_{0}\left(\lambda_{1}\right)+h_{1}\left(\lambda_{2}\right)\left(\lambda-\lambda_{1}\right)+\cdots+h_{n-1}\left(\lambda_{n}\right)\left(\lambda-\lambda_{1}\right) \cdots\left(\lambda-\lambda_{n-1}\right)$ we get (3.2). The uniqueness of decomposition (3.2) follows from the fact that if

$$
\frac{b(\lambda)}{a(\lambda)}=h_{1}(\lambda)+\frac{r_{1}(\lambda)}{a(\lambda)}=h_{2}(\lambda)+\frac{r_{2}(\lambda)}{a(\lambda)},
$$

then

$$
h_{1}(\lambda)-h_{2}(\lambda)=\frac{r_{2}(\lambda)-r_{1}(\lambda)}{a(\lambda)}
$$

where the left hand side is a smooth function on $\mathcal{U}$ and the right hand side is a rational function with singularities at $\lambda_{1}, \lambda_{2}, \ldots, \lambda_{n} \in \mathcal{U}$. Therefore, $h_{1}(\lambda)-h_{2}(\lambda)=0$ and $r_{2}(\lambda)-r_{1}(\lambda)=0$.

We will denote $h(\lambda)$ in the decomposition $(3.2)$ by $\left[\frac{b(\lambda)}{a(\lambda)}\right]_{+}$and $r(\lambda)$ by $b(\lambda) \bmod a(\lambda)$. In practice both these functions can be calculated using the recursive formulas derived in the proof of Lemma 3.1. In a particular case when $b(\lambda)$ is a polynomial or a pure Laurent polynomial we may equivalently use the division algorithms for the division of polynomial by polynomial and the division of pure Laurent polynomial by polynomial. For example if $b(\lambda)=\sum_{k=0}^{m} b_{k} \lambda^{k}$ is a polynomial of order $m$ and $a(\lambda)$ is a polynomial of order $n$ given by (3.1), such that $m \geq n$, $\left[\frac{b(\lambda)}{a(\lambda)}\right]_{+}$is a polynomial part of $\frac{b(\lambda)}{a(\lambda)}$ of order $(m-n)$ and $b(\lambda) \bmod a(\lambda)$ is the reminder of $\frac{b(\lambda)}{a(\lambda)}$, i.e.,

$$
b(\lambda) \bmod a(\lambda)=\operatorname{rem}\left[\frac{b(\lambda)}{a(\lambda)}\right]
$$

being a polynomial of order less than $n$. In the division algorithm we divide $b(\lambda)$ by the highest order term of $a(\lambda)$. On the other hand if $b(\lambda)=c\left(\lambda^{-1}\right)=\sum_{k=1}^{m} c_{k} \lambda^{-k}$ is a pure Laurent polynomial of order $m$ and $a(\lambda)$ is a polynomial of order $n$ given by $(3.1),\left[\frac{c\left(\lambda^{-1}\right)}{a(\lambda)}\right]_{+}$is a pure Laurent polynomial of order $m$ and $c\left(\lambda^{-1}\right) \bmod a(\lambda)$ is the reminder of $\frac{c\left(\lambda^{-1}\right)}{a(\lambda)}$ being again a polynomial of degree less than $n$. In the division algorithm we divide $c\left(\lambda^{-1}\right)$ by the lowest order term of $a(\lambda)$. In the case of arbitrary Laurent polynomial $P\left(\lambda, \lambda^{-1}\right)=b(\lambda)+c\left(\lambda^{-1}\right)$, the division by polynomial $a(\lambda)$ splits onto two parts described above. Note, that for a smooth function $b(\lambda)$ and a polynomial $a(\lambda)$ there holds

$$
b(\lambda)=b(\lambda) \bmod a(\lambda)+a(\lambda)\left[\frac{b(\lambda)}{a(\lambda)}\right]_{+} .
$$

We will consider infinitely many non-equivalent Lax matrices $L \in \mathfrak{s l}(2, \mathbb{R})$ parameterized by smooth functions $g(\lambda)$, everywhere non-zero on the same domain $\mathcal{U}$ as functions $f(\lambda)$ and $\sigma(\lambda)$. The Lax matrices in the canonical representation $(\lambda, \mu)$, parameterized by $g(\lambda)$, are taken in the form

$$
L(\lambda)=\left(\begin{array}{cc}
v(\lambda) & u(\lambda) \\
w(\lambda) & -v(\lambda)
\end{array}\right),
$$

where

$$
u(\lambda)=\prod_{k=1}^{n}\left(\lambda-\lambda_{k}\right)=\sum_{k=0}^{n} \rho_{k} \lambda^{n-k}, \quad \rho_{0} \equiv 1
$$


and

$$
\begin{aligned}
v(\lambda) & =\sum_{i=1}^{n} g\left(\lambda_{i}\right) \mu_{i} \prod_{k \neq i} \frac{\lambda-\lambda_{k}}{\lambda_{i}-\lambda_{k}}=\sum_{i=1}^{n} \frac{u(\lambda)}{\lambda-\lambda_{i}} \frac{g\left(\lambda_{i}\right) \mu_{i}}{\Delta_{i}} \stackrel{(3.6)}{=}-\sum_{k=1}^{n}\left[\sum_{i=1}^{n} \frac{\partial \rho_{k}}{\partial \lambda_{i}} \frac{g\left(\lambda_{i}\right) \mu_{i}}{\Delta_{i}}\right] \lambda^{n-k} \\
& =-\sum_{k=0}^{n-1}\left[\sum_{i=1}^{n} \frac{\partial \rho_{n-k}}{\partial \lambda_{i}} \frac{g\left(\lambda_{i}\right) \mu_{i}}{\Delta_{i}}\right] \lambda^{k} .
\end{aligned}
$$

Notice that $u\left(\lambda_{i}\right)=0, v\left(\lambda_{i}\right)=g\left(\lambda_{i}\right) \mu_{i}$ and

$$
\Delta_{i}=u_{i}(\lambda), \quad u_{i}(\lambda):=\frac{u(\lambda)}{\lambda-\lambda_{i}}=-\frac{\partial u(\lambda)}{\partial \lambda_{i}}=\prod_{k \neq i}\left(\lambda-\lambda_{k}\right)=-\sum_{k=1}^{n} \frac{\partial \rho_{k}}{\partial \lambda_{i}} \lambda^{n-k} .
$$

Moreover,

$$
w(\lambda)=-2 \frac{g^{2}(\lambda)}{f(\lambda)}\left[\frac{F(\lambda, v(\lambda) / g(\lambda))}{u(\lambda)}\right]_{+},
$$

where $F(x, y)=\frac{1}{2} f(x) y^{2}-\sigma(x)$. The function $w(\lambda)$ splits onto kinetic part $w_{E}(\lambda)$ and potential part $w_{V}(\lambda)$ respectively:

$$
w(\lambda)=w_{E}(\lambda)+w_{V}(\lambda)=-\frac{g^{2}(\lambda)}{f(\lambda)}\left[\frac{f(\lambda) v^{2}(\lambda) / g^{2}(\lambda)}{u(\lambda)}\right]_{+}+2 \frac{g^{2}(\lambda)}{f(\lambda)}\left[\frac{\sigma(\lambda)}{u(\lambda)}\right]_{+} .
$$

The main result we state in the following theorem.

Theorem 3.2. For arbitrary everywhere non-zero smooth function $g(\lambda)$, separation curve (1.4) that generates dynamical systems (2.1), is reconstructed as follows

$$
\operatorname{det}[L(\lambda)-g(\lambda) \mu I]=0 \quad \Longleftrightarrow \quad \sigma(\lambda)+\sum_{k=1}^{n} H_{k} \lambda^{n-1}=\frac{1}{2} f(\lambda) \mu^{2} .
$$

Moreover, Lax equations for systems (2.2), parameterized by $g(\lambda)$, take the form

$$
\frac{\mathrm{d}}{\mathrm{d} t_{k}} L(\lambda)=\left[U_{k}(\lambda), L(\lambda)\right]
$$

where the Lax matrices $L(\lambda)$ are defined by (3.3)-(3.7) and

$$
U_{k}(\lambda)=\left[\frac{B_{k}(\lambda)}{u(\lambda)}\right]_{+}, \quad B_{k}(\lambda)=\frac{1}{2} \frac{f(\lambda)}{g(\lambda)}\left[\frac{u(\lambda)}{\lambda^{n-k+1}}\right]_{+} L(\lambda) .
$$

The proof of the above theorem is involved so we present it in the separate section.

As an example let us find explicit formulas for the matrices $L(\lambda)$ and $U_{k}(\lambda)$ written in a coordinate independent way for a general Stäckel system from the Benenti class in the case of two degrees of freedom, i.e., $n=2$. A separation curve of that system has the form

$$
\sigma(\lambda)+H_{1} \lambda+H_{2}=\frac{1}{2} f(\lambda) \mu^{2},
$$

where $f(\lambda)$ and $\sigma(\lambda)$ are smooth functions. According to (2.1) the Hamiltonians in separation coordinates are equal

$$
H_{1}(\lambda, \mu)=\frac{F\left(\lambda_{1}, \mu_{1}\right)-F\left(\lambda_{2}, \mu_{2}\right)}{\lambda_{1}-\lambda_{2}}
$$




$$
\begin{aligned}
& =\frac{1}{2} \frac{f\left(\lambda_{1}\right)}{\lambda_{1}-\lambda_{2}} \mu_{1}^{2}-\frac{1}{2} \frac{f\left(\lambda_{2}\right)}{\lambda_{1}-\lambda_{2}} \mu_{2}^{2}+\frac{\sigma\left(\lambda_{2}\right)-\sigma\left(\lambda_{1}\right)}{\lambda_{1}-\lambda_{2}}, \\
H_{2}(\lambda, \mu) & =\frac{\lambda_{1} F\left(\lambda_{2}, \mu_{2}\right)-\lambda_{2} F\left(\lambda_{1}, \mu_{1}\right)}{\lambda_{1}-\lambda_{2}} \\
& =\frac{1}{2} \frac{\lambda_{1} f\left(\lambda_{2}\right)}{\lambda_{1}-\lambda_{2}} \mu_{2}^{2}-\frac{1}{2} \frac{\lambda_{2} f\left(\lambda_{1}\right)}{\lambda_{1}-\lambda_{2}} \mu_{1}^{2}+\frac{\lambda_{2} \sigma\left(\lambda_{1}\right)-\lambda_{1} \sigma\left(\lambda_{2}\right)}{\lambda_{1}-\lambda_{2}} .
\end{aligned}
$$

The Lax matrix $L(\lambda)$ parameterized by a general everywhere non-zero smooth function $g(\lambda)$ will be equal

$$
L(\lambda)=\left(\begin{array}{cc}
v(\lambda) & u(\lambda) \\
w(\lambda) & -v(\lambda)
\end{array}\right)
$$

where in accordance to (3.4), (3.5), (3.7) and (4.1)

$$
\begin{aligned}
& u(\lambda)=\lambda^{2}+\rho_{1} \lambda+\rho_{2}, \\
& v(\lambda)=v_{1} \lambda+v_{2}, \\
& w(\lambda)=-\frac{v^{2}(\lambda)}{u(\lambda)}+\frac{2 g^{2}(\lambda) \sigma(\lambda)}{f(\lambda) u(\lambda)}+\frac{2 g^{2}(\lambda)}{f(\lambda) u(\lambda)}\left(H_{1} \lambda+H_{2}\right),
\end{aligned}
$$

where in separation coordinates

$$
\begin{aligned}
& \rho_{1}=-\lambda_{1}-\lambda_{2}, \quad \rho_{2}=\lambda_{1} \lambda_{2}, \\
& v_{1}=\frac{g\left(\lambda_{1}\right) \mu_{1}-g\left(\lambda_{2}\right) \mu_{2}}{\lambda_{1}-\lambda_{2}}, \quad v_{2}=\frac{\lambda_{1} g\left(\lambda_{2}\right) \mu_{2}-\lambda_{2} g\left(\lambda_{1}\right) \mu_{1}}{\lambda_{1}-\lambda_{2}} .
\end{aligned}
$$

We find that

$$
U_{k}(\lambda)=\frac{1}{2 u(\lambda)}\left(\begin{array}{cc}
a_{k}(\lambda) & b_{k}(\lambda) \\
c_{k}(\lambda) & -a_{k}(\lambda)
\end{array}\right), \quad k=1,2,
$$

where

$$
\begin{array}{llrl}
a_{1}(\lambda)=\frac{f(\lambda) v(\lambda)}{g(\lambda)}+\left\{u(\lambda), H_{1}\right\}, & a_{2}(\lambda)=\frac{\left(\lambda+\rho_{1}\right) f(\lambda) v(\lambda)}{g(\lambda)}+\left\{u(\lambda), H_{2}\right\}, \\
b_{1}(\lambda)=\frac{f(\lambda) u(\lambda)}{g(\lambda)}, & b_{2}(\lambda)=\frac{\left(\lambda+\rho_{1}\right) f(\lambda) u(\lambda)}{g(\lambda)}, \\
c_{1}(\lambda)=\frac{f(\lambda) w(\lambda)}{g(\lambda)}-2\left\{v(\lambda), H_{1}\right\}, & c_{2}(\lambda)=\frac{\left(\lambda+\rho_{1}\right) f(\lambda) w(\lambda)}{g(\lambda)}-2\left\{v(\lambda), H_{2}\right\} .
\end{array}
$$

Indeed, for a smooth function $b(\lambda)$, with the use of the recursive formulas derived in the proof of Lemma 3.1, we get that

$$
\begin{aligned}
{\left[\frac{b(\lambda)}{u(\lambda)}\right]_{+} } & =\frac{\frac{b(\lambda)-b\left(\lambda_{1}\right)}{\lambda-\lambda_{1}}-\frac{b\left(\lambda_{2}\right)-b\left(\lambda_{1}\right)}{\lambda_{2}-\lambda_{1}}}{\lambda-\lambda_{2}} \\
& =\frac{b(\lambda)}{u(\lambda)}-\frac{1}{u(\lambda)}\left(\frac{b\left(\lambda_{1}\right)-b\left(\lambda_{2}\right)}{\lambda_{1}-\lambda_{2}} \lambda+\frac{\lambda_{1} b\left(\lambda_{2}\right)-\lambda_{2} b\left(\lambda_{1}\right)}{\lambda_{1}-\lambda_{2}}\right)
\end{aligned}
$$

and

$$
\left.\left[\frac{b(\lambda)}{u(\lambda)}\right]_{+}\right|_{\lambda=\lambda_{k}}=(-1)^{k}\left(-\frac{b^{\prime}\left(\lambda_{k}\right)}{\lambda_{1}-\lambda_{2}}+\frac{b\left(\lambda_{1}\right)-b\left(\lambda_{2}\right)}{\left(\lambda_{1}-\lambda_{2}\right)^{2}}\right), \quad k=1,2 .
$$


From (3.13), by putting $b(\lambda)=F(\lambda, v(\lambda) / g(\lambda))$, we can calculate $w\left(\lambda_{1}\right)$ and $w\left(\lambda_{2}\right)$ and then

$$
\begin{aligned}
\frac{f\left(\lambda_{1}\right) w\left(\lambda_{1}\right)}{2\left(\lambda_{1}-\lambda_{2}\right) g\left(\lambda_{1}\right)}-\frac{f\left(\lambda_{2}\right) w\left(\lambda_{2}\right)}{2\left(\lambda_{1}-\lambda_{2}\right) g\left(\lambda_{2}\right)} & =\frac{\partial v_{1}}{\partial \lambda_{1}} \frac{\partial H_{1}}{\partial \mu_{1}}+\frac{\partial v_{1}}{\partial \lambda_{2}} \frac{\partial H_{1}}{\partial \mu_{2}}-\frac{\partial v_{1}}{\partial \mu_{1}} \frac{\partial H_{1}}{\partial \lambda_{1}}-\frac{\partial v_{1}}{\partial \mu_{2}} \frac{\partial H_{1}}{\partial \lambda_{2}} \\
& =\left\{v_{1}, H_{1}\right\} .
\end{aligned}
$$

Using the fact that

$$
\begin{array}{llll}
\frac{\partial v_{2}}{\partial \lambda_{1}}=-\lambda_{2} \frac{\partial v_{1}}{\partial \lambda_{1}}, & \frac{\partial v_{2}}{\partial \lambda_{2}}=-\lambda_{1} \frac{\partial v_{1}}{\partial \lambda_{2}}, & \frac{\partial v_{2}}{\partial \mu_{1}}=-\lambda_{2} \frac{\partial v_{1}}{\partial \mu_{1}}, & \frac{\partial v_{2}}{\partial \mu_{2}}=-\lambda_{1} \frac{\partial v_{1}}{\partial \mu_{2}} \\
\frac{\partial H_{2}}{\partial \lambda_{1}}=-\lambda_{2} \frac{\partial H_{1}}{\partial \lambda_{1}}, & \frac{\partial H_{2}}{\partial \lambda_{2}}=-\lambda_{1} \frac{\partial H_{1}}{\partial \lambda_{2}}, & \frac{\partial H_{2}}{\partial \mu_{1}}=-\lambda_{2} \frac{\partial H_{1}}{\partial \mu_{1}}, & \frac{\partial H_{2}}{\partial \mu_{2}}=-\lambda_{1} \frac{\partial H_{1}}{\partial \mu_{2}}
\end{array}
$$

we also get

$$
\begin{aligned}
& -\frac{\lambda_{2} f\left(\lambda_{1}\right) w\left(\lambda_{1}\right)}{2\left(\lambda_{1}-\lambda_{2}\right) g\left(\lambda_{1}\right)}+\frac{\lambda_{1} f\left(\lambda_{2}\right) w\left(\lambda_{2}\right)}{2\left(\lambda_{1}-\lambda_{2}\right) g\left(\lambda_{2}\right)} \\
& \quad=-\lambda_{2} \frac{\partial v_{1}}{\partial \lambda_{1}} \frac{\partial H_{1}}{\partial \mu_{1}}-\lambda_{1} \frac{\partial v_{1}}{\partial \lambda_{2}} \frac{\partial H_{1}}{\partial \mu_{2}}+\lambda_{2} \frac{\partial v_{1}}{\partial \mu_{1}} \frac{\partial H_{1}}{\partial \lambda_{1}}+\lambda_{1} \frac{\partial v_{1}}{\partial \mu_{2}} \frac{\partial H_{1}}{\partial \lambda_{2}}=\left\{v_{2}, H_{1}\right\}=\left\{v_{1}, H_{2}\right\} \\
& \frac{\lambda_{2}^{2} f\left(\lambda_{1}\right) w\left(\lambda_{1}\right)}{2\left(\lambda_{1}-\lambda_{2}\right) g\left(\lambda_{1}\right)}-\frac{\lambda_{1}^{2} f\left(\lambda_{2}\right) w\left(\lambda_{2}\right)}{2\left(\lambda_{1}-\lambda_{2}\right) g\left(\lambda_{2}\right)} \\
& \quad=\lambda_{2}^{2} \frac{\partial v_{1}}{\partial \lambda_{1}} \frac{\partial H_{1}}{\partial \mu_{1}}+\lambda_{1}^{2} \frac{\partial v_{1}}{\partial \lambda_{2}} \frac{\partial H_{1}}{\partial \mu_{2}}-\lambda_{2}^{2} \frac{\partial v_{1}}{\partial \mu_{1}} \frac{\partial H_{1}}{\partial \lambda_{1}}-\lambda_{1}^{2} \frac{\partial v_{1}}{\partial \mu_{2}} \frac{\partial H_{1}}{\partial \lambda_{2}}=\left\{v_{2}, H_{2}\right\}
\end{aligned}
$$

From (3.12), by putting $b(\lambda)=\frac{f(\lambda) w(\lambda)}{2 g(\lambda)}$ and $b(\lambda)=\frac{\left(\lambda+\rho_{1}\right) f(\lambda) w(\lambda)}{2 g(\lambda)}$, and using (3.14), (3.15), (3.16) and

$$
\left[\frac{u(\lambda)}{\lambda}\right]_{+}=\lambda+\rho_{1}, \quad\left[\frac{u(\lambda)}{\lambda^{2}}\right]_{+}=1
$$

we get formulas for $c_{1}(\lambda)$ and $c_{2}(\lambda)$. Similarly we calculate $a_{1}(\lambda), a_{2}(\lambda), b_{1}(\lambda)$ and $b_{2}(\lambda)$.

Let us notice that for a given Lax representation $(L, U)$, with fixed $g(\lambda)$, there exist infinitely many gauge equivalent Lax representations $\left(L^{\prime}, U^{\prime}\right)$. Actually, let $\Omega$ be a $2 \times 2$ invertible matrix, with matrix elements dependent on phase space coordinates but independent on spectral parameter $\lambda$. Then, for

$$
L^{\prime}=\Omega L \Omega^{-1}, \quad U^{\prime}=\Omega U \Omega^{-1}+\Omega_{t} \Omega^{-1}
$$

one can show that

$$
L_{t}=[U, L] \quad \Longleftrightarrow \quad L_{t}^{\prime}=\left[U^{\prime}, L^{\prime}\right]
$$

and

$$
\operatorname{det}(L-g(\lambda) \mu I)=\operatorname{det}\left(L^{\prime}-g(\lambda) \mu I\right)=0 .
$$

Hence, from the construction, such class of equivalent Lax representations has the same $\lambda$-structure.

\section{Proof of Theorem 3.2}

First, let us prove the following lemma. 
Lemma 4.1. The following equality holds

$$
\sum_{k=1}^{n} H_{k} \lambda^{n-k}=F(\lambda, v(\lambda) / g(\lambda)) \bmod u(\lambda)
$$

for $F(x, y)=\frac{1}{2} f(x) y^{2}-\sigma(x)$ and $H_{k}$ defined by the linear system (1.2).

Proof. In the proof we will use the property that a polynomial of order less than $n$ is uniquely specified by its values at $n$ distinct points. The functions $H_{k}=H_{k}\left(\lambda_{1}, \ldots, \lambda_{n}, \mu_{1}, \ldots, \mu_{n}\right)$ satisfy the equations (1.2)

$$
\sum_{k=1}^{n} H_{k}\left(\lambda_{1}, \ldots, \lambda_{n}, \mu_{1}, \ldots, \mu_{n}\right) \lambda_{i}^{n-k}=F\left(\lambda_{i}, \mu_{i}\right), \quad i=1,2, \ldots, n
$$

For fixed $\lambda_{1}, \ldots, \lambda_{n}, \mu_{1}, \ldots, \mu_{n}$ such that $\lambda_{i} \neq \lambda_{j}$ for $i \neq j$ the expression

$$
\sum_{k=1}^{n} H_{k}\left(\lambda_{1}, \ldots, \lambda_{n}, \mu_{1}, \ldots, \mu_{n}\right) \lambda^{n-k}
$$

is a polynomial in $\lambda$ of order $n-1$, which takes values $F\left(\lambda_{i}, \mu_{i}\right)$ at $\lambda=\lambda_{i}$. On the other hand

$$
F(\lambda, v(\lambda) / g(\lambda)) \bmod u(\lambda)=F(\lambda, v(\lambda) / g(\lambda))-u(\lambda)\left[\frac{F(\lambda, v(\lambda) / g(\lambda))}{u(\lambda)}\right]_{+}
$$

is also a polynomial in $\lambda$ of order $n-1$, which takes the same values $F\left(\lambda_{i}, \mu_{i}\right)$ at $\lambda=\lambda_{i}$, since $u\left(\lambda_{i}\right)=0$ and $v\left(\lambda_{i}\right)=g\left(\lambda_{i}\right) \mu_{i}$. This proves the equality (4.1).

Now we can pass to the proof of formula (3.9).

Proof of (3.9). We calculate that

$$
\begin{aligned}
\operatorname{det}[L(\lambda)-g(\lambda) \mu I]= & \operatorname{det}\left(\begin{array}{cc}
v(\lambda)-g(\lambda) \mu & u(\lambda) \\
w(\lambda) & -v(\lambda)-g(\lambda) \mu
\end{array}\right) \\
= & -(v(\lambda)-g(\lambda) \mu)(v(\lambda)+g(\lambda) \mu)-u(\lambda) w(\lambda) \\
= & -v^{2}(\lambda)+g^{2}(\lambda) \mu^{2}+2 \frac{g^{2}(\lambda)}{f(\lambda)} u(\lambda)\left[\frac{F(\lambda, v(\lambda) / g(\lambda))}{u(\lambda)}\right]_{+} \\
= & -2 \frac{g^{2}(\lambda)}{f(\lambda)}\left(\frac{1}{2} f(\lambda) v^{2}(\lambda) / g^{2}(\lambda)-\sigma(\lambda)-u(\lambda)\left[\frac{F(\lambda, v(\lambda) / g(\lambda))}{u(\lambda)}\right]_{+}\right) \\
& -2 \frac{g^{2}(\lambda)}{f(\lambda)} \sigma(\lambda)+g^{2}(\lambda) \mu^{2} \\
= & -2 \frac{g^{2}(\lambda)}{f(\lambda)}(F(\lambda, v(\lambda) / g(\lambda)) \bmod u(\lambda))+2 \frac{g^{2}(\lambda)}{f(\lambda)}\left(\frac{1}{2} f(\lambda) \mu^{2}-\sigma(\lambda)\right) \\
= & 2 \frac{g^{2}(\lambda)}{f(\lambda)}\left(-\sum_{k=1}^{n} H_{k} \lambda^{n-k}+\frac{1}{2} f(\lambda) \mu^{2}-\sigma(\lambda)\right),
\end{aligned}
$$

where in the last equality we used Lemma 4.1. This proves (3.9).

Now we will show that the Lax equations (3.10) hold. The proof is based on the following lemmas. 
Lemma 4.2. The Poisson bracket of $u(\lambda)$ and $v(\lambda)$ is equal

$$
\begin{aligned}
& \left\{u(\lambda), u\left(\lambda^{\prime}\right)\right\}=0, \quad\left\{v(\lambda), v\left(\lambda^{\prime}\right)\right\}=0 \\
& \left\{u(\lambda), v\left(\lambda^{\prime}\right)\right\}=\left\{u\left(\lambda^{\prime}\right), v(\lambda)\right\}=-\sum_{k=1}^{n}\left(g(\lambda)\left[\frac{u(\lambda)}{\lambda^{n-k+1}}\right]_{+} \bmod u(\lambda)\right) \lambda^{\prime n-k} .
\end{aligned}
$$

Proof. In the proof we will use the property that a polynomial of order less than $n$ is uniquely specified by its values at $n$ distinct points. The first equality in (4.2a) is straightforward. The second equality follows from

$$
\left\{v\left(\lambda_{i}\right), v\left(\lambda_{j}\right)\right\}=\left\{g\left(\lambda_{i}\right) \mu_{i}, g\left(\lambda_{j}\right) \mu_{j}\right\}=0 \quad \text { for } \quad i, j=1,2, \ldots, n .
$$

For the proof of $(4.2 \mathrm{~b})$ note that

$$
\begin{aligned}
\left\{\rho_{k}, v\left(\lambda_{j}\right)\right\} & =\left\{\rho_{k}, g\left(\lambda_{j}\right) \mu_{j}\right\}=(-1)^{k} \sum_{1 \leq l_{1}<l_{2}<\cdots<l_{k} \leq n}\left\{\lambda_{l_{1}} \lambda_{l_{2}} \cdots \lambda_{l_{k}}, g\left(\lambda_{j}\right) \mu_{j}\right\} \\
& =-g\left(\lambda_{j}\right) \sum_{m=0}^{k-1} \lambda_{j}^{m} \rho_{k-m-1}\left\{\lambda_{j}, \mu_{j}\right\}=-g\left(\lambda_{j}\right) \sum_{m=0}^{k-1} \lambda_{j}^{m} \rho_{k-m-1}
\end{aligned}
$$

is a value of the polynomial

$$
\left(-g(\lambda) \sum_{m=0}^{k-1} \lambda^{m} \rho_{k-m-1}\right) \bmod u(\lambda) \quad \text { at } \quad \lambda=\lambda_{j},
$$

since $u\left(\lambda_{j}\right)=0$. Because this polynomial is of order less than $n$ we can write

$$
\left\{\rho_{k}, v(\lambda)\right\}=\left(-g(\lambda) \sum_{m=0}^{k-1} \lambda^{m} \rho_{k-m-1}\right) \bmod u(\lambda)=-g(\lambda)\left[\frac{u(\lambda)}{\lambda^{n-k+1}}\right]_{+} \bmod u(\lambda) .
$$

Thus

$$
\left\{u\left(\lambda^{\prime}\right), v(\lambda)\right\}=\sum_{k=1}^{n}\left\{\rho_{k}, v(\lambda)\right\} \lambda^{\prime n-k}=-\sum_{k=1}^{n}\left(g(\lambda)\left[\frac{u(\lambda)}{\lambda^{n-k+1}}\right]_{+} \bmod u(\lambda)\right) \lambda^{\prime n-k},
$$

which proves the second equality in $(4.2 \mathrm{~b})$. For the proof of the first equality in $(4.2 \mathrm{~b})$ we calculate that

$$
\begin{aligned}
\left\{u\left(\lambda^{\prime}\right), v(\lambda)\right\} & =\left\{\prod_{i=1}^{n}\left(\lambda^{\prime}-\lambda_{i}\right), \sum_{l=1}^{n} g\left(\lambda_{l}\right) \mu_{l} \prod_{j \neq l} \frac{\lambda-\lambda_{j}}{\lambda_{l}-\lambda_{j}}\right\} \\
& =\sum_{l=1}^{n} g\left(\lambda_{l}\right)\left\{\prod_{i=1}^{n}\left(\lambda^{\prime}-\lambda_{i}\right), \mu_{l}\right\} \prod_{j \neq l} \frac{\lambda-\lambda_{j}}{\lambda_{l}-\lambda_{j}} \\
& =-\sum_{l=1}^{n} g\left(\lambda_{l}\right)\left\{\lambda_{l}, \mu_{l}\right\} \prod_{j \neq l} \frac{\left(\lambda^{\prime}-\lambda_{j}\right)\left(\lambda-\lambda_{j}\right)}{\lambda_{l}-\lambda_{j}}=\left\{u(\lambda), v\left(\lambda^{\prime}\right)\right\} .
\end{aligned}
$$

Lemma 4.3. Let $q(\lambda)$ be a polynomial of order $n$ and $p(\lambda)$ a smooth function defined on a domain containing all roots of $q(\lambda)$, then

$$
\sum_{k=1}^{n} \lambda^{\prime n-k} p(\lambda)\left[\lambda^{-n+k-1} q(\lambda)\right]_{+} \bmod q(\lambda)=\sum_{k=1}^{n} \lambda^{n-k} p\left(\lambda^{\prime}\right)\left[\lambda^{\prime-n+k-1} q\left(\lambda^{\prime}\right)\right]_{+} \bmod q\left(\lambda^{\prime}\right) .
$$


Proof. We have that

$$
p(\lambda)\left[\lambda^{-n+k-1} q(\lambda)\right]_{+} \bmod q(\lambda)=r(\lambda)\left[\lambda^{-n+k-1} q(\lambda)\right]_{+} \bmod q(\lambda),
$$

where $r(\lambda)=p(\lambda) \bmod q(\lambda)$. Since $\left[\frac{r(\lambda)}{q(\lambda)}\right]_{+}=0$ it further follows that

$$
\begin{aligned}
p(\lambda)\left[\lambda^{-n+k-1} q(\lambda)\right]_{+} \bmod q(\lambda) & =r(\lambda)\left[\lambda^{-n+k-1} q(\lambda)\right]_{+}-q(\lambda)\left[\frac{r(\lambda)}{q(\lambda)}\left[\lambda^{-n+k-1} q(\lambda)\right]_{+}\right]_{+} \\
& =r(\lambda)\left[\lambda^{-n+k-1} q(\lambda)\right]_{+}-q(\lambda)\left[\frac{r(\lambda)}{q(\lambda)} \lambda^{-n+k-1} q(\lambda)\right]_{+} \\
& =r(\lambda)\left[\lambda^{-n+k-1} q(\lambda)\right]_{+}-q(\lambda)\left[\lambda^{-n+k-1} r(\lambda)\right]_{+} .
\end{aligned}
$$

Using this equality we get

$$
\begin{aligned}
\sum_{k=1}^{n} \lambda^{\prime n-k} p(\lambda)\left[\lambda^{-n+k-1} q(\lambda)\right]_{+} \bmod q(\lambda) & \\
& =\sum_{k=1}^{n} \lambda^{\prime n-k}\left(r(\lambda)\left[\lambda^{-n+k-1} q(\lambda)\right]_{+}-q(\lambda)\left[\lambda^{-n+k-1} r(\lambda)\right]_{+}\right) \\
& =\sum_{k=1}^{n} \lambda^{n-k}\left(r\left(\lambda^{\prime}\right)\left[\lambda^{\prime-n+k-1} q\left(\lambda^{\prime}\right)\right]_{+}-q\left(\lambda^{\prime}\right)\left[\lambda^{\prime-n+k-1} r\left(\lambda^{\prime}\right)\right]_{+}\right) \\
& =\sum_{k=1}^{n} \lambda^{n-k} p\left(\lambda^{\prime}\right)\left[\lambda^{\prime-n+k-1} q\left(\lambda^{\prime}\right)\right]_{+} \bmod q\left(\lambda^{\prime}\right),
\end{aligned}
$$

where the second equality is easily proven by expanding the polynomials.

Lemma 4.4. The action of Hamiltonian vector fields $X_{k}=\left\{\cdot, H_{k}\right\}$ on $u(\lambda)$ and $v(\lambda)$ is equal

$$
\begin{aligned}
& X_{k} u(\lambda)=-\frac{\partial F}{\partial y}(\lambda, v(\lambda) / g(\lambda))\left[\frac{u(\lambda)}{\lambda^{n-k+1}}\right]_{+} \bmod u(\lambda), \\
& X_{k} v(\lambda)=-g(\lambda)\left[\frac{F(\lambda, v(\lambda) / g(\lambda))}{u(\lambda)}\right]_{+}\left[\frac{u(\lambda)}{\lambda^{n-k+1}}\right]_{+} \bmod u(\lambda),
\end{aligned}
$$

where $F(x, y)=\frac{1}{2} f(x) y^{2}-\sigma(x)$.

Proof. Using Lemmas 4.1, 4.2 and 4.3 we calculate that

$$
\begin{aligned}
\sum_{k=1}^{n}\left\{u\left(\lambda^{\prime}\right), H_{k}\right\} \lambda^{n-k} & =\left\{u\left(\lambda^{\prime}\right), F(\lambda, v(\lambda) / g(\lambda)) \bmod u(\lambda)\right\} \\
& =\sum_{i=1}^{n} \frac{\partial u\left(\lambda^{\prime}\right)}{\partial \lambda_{i}} \frac{\partial F}{\partial y}(\lambda, v(\lambda) / g(\lambda)) \frac{1}{g(\lambda)} \frac{\partial v(\lambda)}{\partial \mu_{i}} \bmod u(\lambda) \\
& =\left\{u\left(\lambda^{\prime}\right), v(\lambda)\right\} \frac{1}{g(\lambda)} \frac{\partial F}{\partial y}(\lambda, v(\lambda) / g(\lambda)) \bmod u(\lambda) \\
& =-\sum_{k=1}^{n}\left(\frac{\partial F}{\partial y}(\lambda, v(\lambda) / g(\lambda))\left[\frac{u(\lambda)}{\lambda^{n-k+1}}\right]_{+} \bmod u(\lambda)\right) \lambda^{\prime n-k} \\
& =-\sum_{k=1}^{n}\left(\frac{\partial F}{\partial y}\left(\lambda^{\prime}, v\left(\lambda^{\prime}\right) / g\left(\lambda^{\prime}\right)\right)\left[\frac{u\left(\lambda^{\prime}\right)}{\lambda^{\prime n-k+1}}\right]_{+} \bmod u\left(\lambda^{\prime}\right)\right) \lambda^{n-k} .
\end{aligned}
$$


By comparing the coefficients of $\lambda^{n-k}$ on the left and right hand side of the above equality we get equation (4.3a). For the proof of (4.3b) we first calculate

$$
\begin{aligned}
& \frac{\partial}{\partial \lambda_{i}}(F(\lambda, v(\lambda) / g(\lambda)) \bmod u(\lambda))=\frac{\partial}{\partial \lambda_{i}}\left(F(\lambda, v(\lambda) / g(\lambda))-u(\lambda)\left[\frac{F(\lambda, v(\lambda) / g(\lambda))}{u(\lambda)}\right]_{+}\right) \\
&=\frac{1}{g(\lambda)} \frac{\partial F}{\partial y}(\lambda, v(\lambda) / g(\lambda)) \frac{\partial v(\lambda)}{\partial \lambda_{i}}-\frac{\partial u(\lambda)}{\partial \lambda_{i}}\left[\frac{F(\lambda, v(\lambda) / g(\lambda))}{u(\lambda)}\right]_{+} \\
&-u(\lambda)\left[\frac{1}{g(\lambda) u(\lambda)} \frac{\partial F}{\partial y}(\lambda, v(\lambda) / g(\lambda)) \frac{\partial v(\lambda)}{\partial \lambda_{i}}-\frac{F(\lambda, v(\lambda) / g(\lambda))}{u^{2}(\lambda)} \frac{\partial u(\lambda)}{\partial \lambda_{i}}\right]_{+} \\
&= \frac{1}{g(\lambda)} \frac{\partial F}{\partial y}(\lambda, v(\lambda) / g(\lambda)) \frac{\partial v(\lambda)}{\partial \lambda_{i}} \bmod u(\lambda)-\frac{\partial u(\lambda)}{\partial \lambda_{i}}\left[\frac{F(\lambda, v(\lambda) / g(\lambda))}{u(\lambda)}\right]_{+} \\
&+u(\lambda)\left[\frac{F(\lambda, v(\lambda) / g(\lambda))}{u^{2}(\lambda)} \frac{\partial u(\lambda)}{\partial \lambda_{i}}\right]_{+} .
\end{aligned}
$$

Since $\left[\frac{1}{u(\lambda)} \frac{\partial u(\lambda)}{\partial \lambda_{i}}\right]_{+}=0$ we can write

$$
\begin{aligned}
{\left[\frac{1}{u(\lambda)} \frac{\partial u(\lambda)}{\partial \lambda_{i}} \frac{F(\lambda, v(\lambda) / g(\lambda))}{u(\lambda)}\right]_{+} } & =\left[\frac{1}{u(\lambda)} \frac{\partial u(\lambda)}{\partial \lambda_{i}}\left[\frac{F(\lambda, v(\lambda) / g(\lambda))}{u(\lambda)}\right]_{+}\right]_{+} \\
& =\frac{\partial u(\lambda)}{\partial \lambda_{i}}\left[\frac{1}{u(\lambda)}\left[\frac{F(\lambda, v(\lambda) / g(\lambda))}{u(\lambda)}\right]_{+}\right]_{+}
\end{aligned}
$$

and we get

$$
\begin{aligned}
\frac{\partial}{\partial \lambda_{i}}(F(\lambda, v(\lambda) / g(\lambda)) \bmod u(\lambda))= & \frac{1}{g(\lambda)} \frac{\partial v(\lambda)}{\partial \lambda_{i}} \frac{\partial F}{\partial y}(\lambda, v(\lambda) / g(\lambda)) \bmod u(\lambda) \\
& -\frac{\partial u(\lambda)}{\partial \lambda_{i}}\left[\frac{F(\lambda, v(\lambda) / g(\lambda))}{u(\lambda)}\right]_{+} \bmod u(\lambda) .
\end{aligned}
$$

Using this equality and Lemmas 4.1, 4.2 and 4.3 we have

$$
\begin{aligned}
\sum_{k=1}^{n}\left\{v\left(\lambda^{\prime}\right), H_{k}\right\} \lambda^{n-k}= & \left\{v\left(\lambda^{\prime}\right), F(\lambda, v(\lambda) / g(\lambda)) \bmod u(\lambda)\right\} \\
= & \sum_{i=1}^{n}\left(\frac{\partial v\left(\lambda^{\prime}\right)}{\partial \lambda_{i}} \frac{\partial v(\lambda)}{\partial \mu_{i}} \frac{1}{g(\lambda)} \frac{\partial F}{\partial y}(\lambda, v(\lambda) / g(\lambda)) \bmod u(\lambda)\right. \\
& -\frac{\partial v\left(\lambda^{\prime}\right)}{\partial \mu_{i}} \frac{\partial v(\lambda)}{\partial \lambda_{i}} \frac{1}{g(\lambda)} \frac{\partial F}{\partial y}(\lambda, v(\lambda) / g(\lambda)) \bmod u(\lambda) \\
& \left.+\frac{\partial v\left(\lambda^{\prime}\right)}{\partial \mu_{i}} \frac{\partial u(\lambda)}{\partial \lambda_{i}}\left[\frac{F(\lambda, v(\lambda) / g(\lambda))}{u(\lambda)}\right]_{+} \bmod u(\lambda)\right) \\
= & \left\{v\left(\lambda^{\prime}\right), v(\lambda)\right\} \frac{1}{g(\lambda)} \frac{\partial F}{\partial y}(\lambda, v(\lambda) / g(\lambda)) \bmod u(\lambda) \\
& +\left\{u(\lambda), v\left(\lambda^{\prime}\right)\right\}\left[\frac{F(\lambda, v(\lambda) / g(\lambda))}{u(\lambda)}\right]_{+} \bmod u(\lambda) \\
= & -\sum_{k=1}^{n}\left(g(\lambda)\left[\frac{F(\lambda, v(\lambda) / g(\lambda))}{u(\lambda)}\right]_{+}\left[\frac{u(\lambda)}{\lambda^{n-k+1}}\right]_{+} \bmod u(\lambda)\right) \lambda^{\prime n-k} \\
= & -\sum_{k=1}^{n}\left(g\left(\lambda^{\prime}\right)\left[\frac{F\left(\lambda^{\prime}, v\left(\lambda^{\prime}\right) / g\left(\lambda^{\prime}\right)\right)}{u\left(\lambda^{\prime}\right)}\right]_{+}\left[\frac{u\left(\lambda^{\prime}\right)}{\lambda^{\prime n-k+1}}\right]_{+} \bmod u\left(\lambda^{\prime}\right)\right) \lambda^{n-k} .
\end{aligned}
$$

This proves equation (4.3b). 
Proof of (3.10). The equations (4.3) can be rewritten in the form

$$
\begin{aligned}
& X_{k} u(\lambda)=-\frac{f(\lambda)}{g(\lambda)} v(\lambda)\left[\frac{u(\lambda)}{\lambda^{n-k+1}}\right]_{+}+u(\lambda)\left[\frac{f(\lambda)}{g(\lambda)} \frac{v(\lambda)}{u(\lambda)}\left[\frac{u(\lambda)}{\lambda^{n-k+1}}\right]_{+}\right]_{+}, \\
& X_{k} v(\lambda)=\frac{1}{2} \frac{f(\lambda)}{g(\lambda)} w(\lambda)\left[\frac{u(\lambda)}{\lambda^{n-k+1}}\right]_{+}-\frac{1}{2} u(\lambda)\left[\frac{f(\lambda)}{g(\lambda)} \frac{w(\lambda)}{u(\lambda)}\left[\frac{u(\lambda)}{\lambda^{n-k+1}}\right]_{+}\right]_{+} .
\end{aligned}
$$

We can now compute $X_{k} w(\lambda)$

$$
\begin{aligned}
X_{k} w(\lambda) & =-2 \frac{g^{2}(\lambda)}{f(\lambda)} X_{k}\left[\frac{F(\lambda, v(\lambda) / g(\lambda))}{u(\lambda)}\right]_{+} \\
& =-2 \frac{g^{2}(\lambda)}{f(\lambda)}\left[\frac{f(\lambda)}{g^{2}(\lambda)} \frac{v(\lambda)}{u(\lambda)} X_{k} v(\lambda)\right]_{+}+2 \frac{g^{2}(\lambda)}{f(\lambda)}\left[\frac{F(\lambda, v(\lambda) / g(\lambda))}{u(\lambda)} \frac{X_{k} u(\lambda)}{u(\lambda)}\right]_{+} .
\end{aligned}
$$

Since $\left[\frac{X_{k} u(\lambda)}{u(\lambda)}\right]_{+}=0$ we can write

$$
\begin{aligned}
{\left[\frac{F(\lambda, v(\lambda) / g(\lambda))}{u(\lambda)} \frac{X_{k} u(\lambda)}{u(\lambda)}\right]_{+} } & =\left[\left[\frac{F(\lambda, v(\lambda) / g(\lambda))}{u(\lambda)}\right]_{+} \frac{X_{k} u(\lambda)}{u(\lambda)}\right]_{+} \\
& =-\frac{1}{2}\left[\frac{f(\lambda)}{g^{2}(\lambda)} \frac{w(\lambda)}{u(\lambda)} X_{k} u(\lambda)\right]_{+} .
\end{aligned}
$$

By (4.4) and (4.5) we have

$$
\begin{aligned}
X_{k} w(\lambda)= & -2 \frac{g^{2}(\lambda)}{f(\lambda)}\left[\frac{f(\lambda)}{g^{2}(\lambda)} \frac{v(\lambda)}{u(\lambda)} X_{k} v(\lambda)\right]_{+}-\frac{g^{2}(\lambda)}{f(\lambda)}\left[\frac{f(\lambda)}{g^{2}(\lambda)} \frac{w(\lambda)}{u(\lambda)} X_{k} u(\lambda)\right]_{+} \\
= & \frac{g^{2}(\lambda)}{f(\lambda)}\left[\frac{f(\lambda)}{g^{2}(\lambda)} v(\lambda)\left[\frac{f(\lambda)}{g(\lambda)} \frac{w(\lambda)}{u(\lambda)}\left[\frac{u(\lambda)}{\lambda^{n-k+1}}\right]_{+}\right]_{+}\right]_{+} \\
& -\frac{g^{2}(\lambda)}{f(\lambda)}\left[\frac{f(\lambda)}{g^{2}(\lambda)} w(\lambda)\left[\frac{f(\lambda)}{g(\lambda)} \frac{v(\lambda)}{u(\lambda)}\left[\frac{u(\lambda)}{\lambda^{n-k+1}}\right]_{+}\right]_{+}\right]_{+}\left[\frac{f(\lambda)}{g(\lambda)} \frac{v(\lambda)}{u(\lambda)}\left[\frac{u(\lambda)}{\lambda^{n-k+1}}\right]_{+}\right]_{+} v(\lambda)\left[\frac{f(\lambda)}{g(\lambda)} \frac{w(\lambda)}{u(\lambda)}\left[\frac{u(\lambda)}{\lambda^{n-k+1}}\right]_{+}\right]_{+} \\
= & -w(\lambda)
\end{aligned}
$$

From (4.4) and (4.6) we get

$$
\frac{\mathrm{d}}{\mathrm{d} t_{k}} L(\lambda)=X_{k} L(\lambda)=\left[U_{k}(\lambda), L(\lambda)\right]
$$

\section{Lax representation in Viète coordinates}

Separation coordinates $\left(\lambda_{i}, \mu_{i}\right)_{i=1, \ldots, n}$ are important from the point of view of integrability of considered systems, but not practical for our purpose, as for $n>2$, matrix elements of any Lax pair $\left(L, U_{k}\right)$ contain in any case a complicated rational functions. So, let us express considered systems and their Lax representations in so called Viète coordinates

$$
q_{i}=\rho_{i}(\lambda), \quad p_{i}=-\sum_{k=1}^{n} \frac{\lambda_{k}^{n-i} \mu_{k}}{\Delta_{k}}, \quad i=1, \ldots, n .
$$

The advantage of such coordinates relies on the fact that for $\sigma(\lambda), f(\lambda)$ and $g(\lambda)$ of polynomial form, all Hamiltonians and Lax matrix elements are polynomial functions of Viète coordinates 
as well. Here, just for the simplicity of formulas, we will consider the particular class of systems where $f(\lambda)=\lambda^{m}, g(\lambda)=\lambda^{r}$ and $\sigma(\lambda)=\lambda^{\gamma}, m, r, \gamma \in \mathbb{Z}$.

The Hamiltonians (2.1) take the form

$$
H_{j}=\sum_{i, k}\left(K_{j} G_{m}\right)^{i k} p_{j} p_{k}+V_{j}^{(\gamma)}(q)
$$

where

$$
\left(G_{m}\right)^{i k}=-\sum_{l=0}^{k-1} q_{k-l-1} V_{i}^{(m+l)}(q), \quad\left(K_{j}\right)_{k}^{i}=-\sum_{l=0}^{j-1} q_{l-j-1} V_{i}^{(n+l-k)}(q)
$$

and basic potentials $V^{(\gamma)}$ in $q$ coordinates are generated by the recursion matrix

$$
R=\left(\begin{array}{cccc}
-q_{1} & 1 & 0 & 0 \\
\vdots & 0 & \ddots & 0 \\
\vdots & 0 & 0 & 1 \\
-q_{n} & 0 & 0 & 0
\end{array}\right), \quad R^{-1}=\left(\begin{array}{cccc}
0 & 0 & 0 & -\frac{1}{q_{n}} \\
1 & 0 & 0 & \vdots \\
0 & \ddots & 0 & \vdots \\
0 & 0 & 1 & -\frac{q_{n-1}}{q_{n}}
\end{array}\right)
$$

For Lax representation, we get immediately

$$
u(\lambda ; q)=\sum_{k=0}^{n} q_{k} \lambda^{n-k}, \quad q_{0} \equiv 1 .
$$

From (2.4) and (5.1) it follows that

$$
\sum_{k=1}^{n} \frac{\lambda_{k}^{n+r-i} \mu_{k}}{\Delta_{k}}=-\sum_{j=1}^{n} V_{j}^{(n+r-i)} \sum_{k=1}^{n} \frac{\lambda_{k}^{n-j} \mu_{k}}{\Delta_{k}}=\sum_{j=1}^{n} V_{j}^{(n+r-i)} p_{j} .
$$

So,

$$
\begin{aligned}
v(\lambda ; q, p) & =-\sum_{k=1}^{n}\left(\sum_{i=1}^{n} \frac{\partial \rho_{k}}{\partial \lambda_{i}} \frac{\lambda_{i}^{r} \mu_{i}}{\Delta_{i}}\right) \lambda^{n-k}=\sum_{k=1}^{n}\left[\sum_{i=1}^{n}\left(\sum_{s=0}^{k-1} \rho_{s} \frac{\lambda_{i}^{r+k-s-1} \mu_{i}}{\Delta_{i}}\right)\right] \lambda^{n-k} \\
& =\sum_{k=1}^{n}\left[\sum_{s=0}^{k-1} q_{s}\left(\sum_{j=1}^{n} V_{j}^{(r+k-s-1)} p_{j}\right)\right] \lambda^{n-k},
\end{aligned}
$$

where we used the identity

$$
\frac{\partial \rho_{k}}{\partial \lambda_{i}}=-\sum_{s=0}^{k-1} \rho_{s} \lambda_{i}^{k-s-1}
$$

Notice that in particular for $r=0$

$$
v(\lambda ; q, p)=-\sum_{k=1}^{n}\left[\sum_{j=0}^{k-1} q_{k-j-1} p_{n-j}\right] \lambda^{n-k} .
$$

Thus, the substitutions (5.2), (5.3), (3.8) and (3.11) in $L(\lambda ; q, p)$ and $U_{k}(\lambda ; q, p)$ lead to Lax equations (3.10), for $f(\lambda)=\lambda^{m}, g(\lambda)=\lambda^{r}$, written in canonical $(q, p)$ coordinates.

Besides, when $f(\lambda)$ is a polynomial of order less or equal $n$, the contravariant metric tensor

$$
G=\operatorname{diag}\left(\frac{f\left(\lambda_{1}\right)}{\Delta_{1}}, \ldots, \frac{f\left(\lambda_{n}\right)}{\Delta_{n}}\right)
$$

defined by $E_{1}$ in (2.3) is flat, so one can pass from Viète coordinates to various admissible flat coordinates [9]. 


\section{Examples}

Example 6.1. Our first example is a system described by a separation curve of the canonical form

$$
\lambda^{5}+H_{1} \lambda^{2}+H_{2} \lambda+H_{3}=\frac{1}{2} \mu^{2}
$$

i.e., $n=3$ and $f(\lambda)=1$. This is the case for which there exist flat coordinates [6], related to Viète coordinates by

$$
\begin{aligned}
& q_{1}=x_{1}, \quad q_{2}=x_{2}+\frac{1}{4} x_{1}^{2}, \quad q_{3}=x_{3}+\frac{1}{2} x_{1} x_{2}, \\
& p_{1}=y_{1}-\frac{1}{2} x_{1} y_{2}+\left(\frac{1}{4} x_{1}^{2}-\frac{1}{2} x_{2}\right) y_{3}, \quad p_{2}=y_{2}-\frac{1}{2} x_{1} y_{3}, \quad p_{3}=y_{3} .
\end{aligned}
$$

In flat coordinates Hamiltonians are

$$
\begin{aligned}
H_{1}= & \frac{1}{2} y_{2}^{2}+y_{1} y_{3}+\frac{1}{2} x_{1}^{3}-\frac{3}{2} x_{1} x_{2}+x_{3}, \\
H_{2}= & y_{1} y_{2}+\frac{1}{2} x_{1} y_{2}^{2}-\frac{1}{2} x_{3} y_{3}^{2}+\frac{1}{2} x_{1} y_{1} y_{3}-\frac{1}{2} x_{2} y_{2} y_{3}+\frac{3}{16} x_{1}^{4}-x_{1} x_{3}-x_{2}^{2}, \\
H_{3}= & \frac{1}{2} y_{1}^{2}+\frac{1}{8} x_{1}^{2} y_{2}^{2}+\frac{1}{8} x_{2}^{2} y_{3}^{2}+\frac{1}{2} x_{1} y_{1} y_{2}+\frac{1}{2} x_{2} y_{1} y_{3}-\left(\frac{1}{4} x_{1} x_{2}+x_{3}\right) y_{2} y_{3} \\
& +\frac{3}{4} x_{1}^{2} x_{3}+\frac{3}{8} x_{1}^{3} x_{2}-x_{2} x_{3}-\frac{1}{2} x_{1} x_{2}^{2}
\end{aligned}
$$

and Lax representation for $g(\lambda)=1$ takes the form

$$
\begin{aligned}
& L=\left(\begin{array}{cc}
-y_{3} \lambda^{2}-\left(y_{2}+\frac{1}{2} x_{1} y_{3}\right) \lambda & \lambda^{3}+x_{1} \lambda^{2}+\left(\frac{1}{4} x_{1}^{2}+x_{2}\right) \lambda \\
-y_{1}-\frac{1}{2} x_{1} y_{2}-\frac{1}{2} x_{2} y_{3} & +x_{3}+\frac{1}{2} x_{1} x_{2} \\
2 \lambda^{2}-\left(y_{3}^{2}+2 x_{1}\right) \lambda & y_{3} \lambda^{2}+\left(y_{2}+\frac{1}{2} x_{1} y_{3}\right) \lambda \\
-2 y_{2} y_{3}+\frac{3}{2} x_{1}^{2}-2 x_{2} & +y_{1}+\frac{1}{2} x_{1} y_{2}+\frac{1}{2} x_{2} y_{3}
\end{array}\right), \\
& U_{1}=\left(\begin{array}{cc}
0 & \frac{1}{2} \\
0 & 0
\end{array}\right), \quad U_{2}=\left(\begin{array}{cc}
-\frac{1}{2} y_{3} & \frac{1}{2} \lambda+\frac{1}{2} x_{1} \\
1 & \frac{1}{2} y_{3}
\end{array}\right), \\
& U_{3}=\left(\begin{array}{cc}
-\frac{1}{2} y_{3} \lambda-\frac{1}{2} y_{2}-\frac{1}{4} x_{1} y_{3} & \frac{1}{2} \lambda^{2}+\frac{1}{2} x_{1} \lambda+\frac{1}{8} x_{1}^{2}+\frac{1}{2} x_{2} \\
\lambda-\frac{1}{2} y_{3}^{2}-x_{1} & \frac{1}{2} y_{3} \lambda+\frac{1}{2} y_{2}+\frac{1}{4} x_{1} y_{3}
\end{array}\right) .
\end{aligned}
$$

Example 6.2. Our second example is a system described by a separation curve of the canonical form

$$
H_{1} \lambda+H_{2}=\frac{1}{2} \lambda \mu^{2}+\lambda^{4}
$$

i.e., $n=2$ and $f(\lambda)=\lambda$. This is one of the integrable cases of the Hènon-Heiles system. Actually, in Cartesian coordinates, related to Viète coordinates by

$$
q_{1}=-x_{1}, \quad q_{2}=-\frac{1}{4} x_{2}^{2}, \quad p_{1}=-y_{1}, \quad p_{2}=-\frac{2 y_{2}}{x_{2}},
$$

both Hamiltonians are

$$
H_{1}=\frac{1}{2} y_{1}^{2}+\frac{1}{2} y_{2}^{2}+x_{1}^{3}+\frac{1}{2} x_{1} x_{2}^{2},
$$




$$
H_{2}=\frac{1}{2} x_{2} y_{1} y_{2}-\frac{1}{2} x_{1} y_{2}^{2}+\frac{1}{4} x_{1}^{2} x_{2}^{2}+\frac{1}{16} x_{2}^{4} .
$$

Lax representation for $g(\lambda)=1$ takes the form

$$
\begin{aligned}
& L=\left(\begin{array}{cc}
\frac{2 y_{2}}{x_{2}} \lambda+y_{1}-\frac{2 x_{1} y_{2}}{x_{2}} & \lambda^{2}-x_{1} \lambda-\frac{1}{4} x_{2}^{2} \\
-2 \lambda-\left(\frac{4 y_{2}^{2}}{x_{2}^{2}}+2 x_{1}\right)+\left(\frac{4 x_{1} y_{2}^{2}}{x_{2}^{2}}-\frac{4 y_{1} y_{2}}{x_{2}}-2 x_{1}^{2}-\frac{1}{2} x_{2}^{2}\right) \lambda^{-1} & -\frac{2 y_{2}}{x_{2}} \lambda-y_{1}+\frac{2 x_{1} y_{2}}{x_{2}}
\end{array}\right), \\
& U_{1}=\left(\begin{array}{cc}
\frac{y_{2}}{x_{2}} & \frac{1}{2} \lambda \\
-1 & -\frac{y_{2}}{x_{2}}
\end{array}\right), \quad U_{2}=\left(\begin{array}{cc}
\frac{y_{2}}{x_{2}} \lambda-\frac{x_{1} y_{2}}{x_{2}}+\frac{1}{2} y_{1} & \frac{1}{2} \lambda^{2}-\frac{1}{2} x_{1} \lambda \\
-\lambda-\frac{2 y_{2}^{2}}{x_{2}^{2}}-x_{1} & -\frac{y_{2}}{x_{2}} \lambda+\frac{x_{1} y_{2}}{x_{2}}-\frac{1}{2} y_{1}
\end{array}\right) .
\end{aligned}
$$

Lax representation for $g(\lambda)=\lambda$ is

$$
\begin{aligned}
& L=\left(\begin{array}{cc}
y_{1} \lambda+\frac{1}{2} x_{2} y_{2} & \lambda^{2}-x_{1} \lambda-\frac{1}{4} x_{2}^{2} \\
-2 \lambda^{3}-2 x_{1} \lambda^{2}-\left(2 x_{1}^{2}+\frac{1}{2} x_{2}^{2}\right) \lambda+y_{2}^{2} & -y_{1} \lambda-\frac{1}{2} x_{2} y_{2}
\end{array}\right), \\
& U_{1}=\left(\begin{array}{cc}
0 & \frac{1}{2} \\
-\lambda-2 x_{1} & 0
\end{array}\right), \quad U_{2}=\left(\begin{array}{cc}
\frac{1}{2} y_{1} & \frac{1}{2} \lambda-\frac{1}{2} x_{1} \\
-\lambda^{2}-x_{1} \lambda-x_{1}^{2}-\frac{1}{2} x_{2}^{2} & -\frac{1}{2} y_{1}
\end{array}\right),
\end{aligned}
$$

while Lax representation for $g(\lambda)=\lambda^{2}$ is of the form

$$
\begin{aligned}
L & =\left(\begin{array}{cc}
\left(x_{1} y_{1}+\frac{1}{2} x_{2} y_{2}\right) \lambda+\frac{1}{4} x_{2}^{2} y_{1} & \lambda^{2}-x_{1} \lambda-\frac{1}{4} x_{2}^{2} \\
-2 \lambda^{5}-2 x_{1} \lambda^{4}-\left(2 x_{1}^{2}+\frac{1}{2} x_{2}^{2}\right) \lambda^{3}+\left(y_{1}^{2}+y_{2}^{2}\right) \lambda^{2} & -\left(x_{1} y_{1}+\frac{1}{2} x_{2} y_{2}\right) \lambda-\frac{1}{4} x_{2}^{2} y_{1}
\end{array}\right), \\
+y_{1}\left(x_{1} y_{1}+x_{2} y_{2}\right) \lambda+\frac{1}{2} x_{1} y_{1}^{2} & \\
-\frac{1}{2} y_{1} \lambda^{-1} & \frac{1}{2} \lambda^{-1} \\
U_{1} & =\left(\begin{array}{cc}
2 & \\
-\lambda^{2}-2 x_{1} \lambda-\left(3 x_{1}^{2}+\frac{1}{2} x_{2}^{2}\right)-\frac{1}{2} y_{1}^{2} \lambda^{-1} & \frac{1}{2} y_{1} \lambda^{-1}
\end{array}\right), \\
\frac{1}{2} x_{1} y_{1} \lambda^{-1} & \frac{1}{2}-\frac{1}{2} x_{1} \lambda^{-1} \\
U_{2} & =\left(\begin{array}{cr}
\lambda^{3}-x_{1} \lambda^{2}-\left(x_{1}^{2}+\frac{1}{2} x_{2}^{2}\right) \lambda+\frac{1}{2}\left(y_{1}^{2}+y_{2}^{2}-x_{1} x_{2}^{2}\right)+\frac{1}{2} x_{1} y_{1}^{2} \lambda^{-1} & -\frac{1}{2} x_{1} y_{1} \lambda^{-1}
\end{array}\right) .
\end{aligned}
$$

Lax representations for $g(\lambda)=1$ and $g(\lambda)=\lambda^{2}$ are new one, at least to the knowledge of authors, while that for $g(\lambda)=\lambda$ is well known (see for example [12] or [11]).

Example 6.3. Our last example is a system described by a separation curve of the canonical form

$$
\lambda^{-2}+H_{1} \lambda+H_{2}=\frac{1}{2} \lambda^{-1} \mu^{2},
$$

i.e., $n=2$ and $f(\lambda)=\lambda^{-1}$. Contrary to the previous cases the metric defined by $H_{1}$ is non-flat. In Viète coordinates

$$
\begin{aligned}
& H_{1}=-\frac{1}{2} \frac{p_{1}^{2}}{q_{2}}-\frac{q_{1} p_{1} p_{2}}{q_{2}}+\frac{1}{2}\left(1-\frac{q_{1}^{2}}{q_{2}}\right) p_{2}^{2}-\frac{q_{1}}{q_{2}^{2}}, \\
& H_{2}=-\frac{1}{2} \frac{q_{1} p_{1}^{2}}{q_{2}}+\left(1-\frac{q_{1}^{2}}{q_{2}}\right) p_{1} p_{2}+\left(q_{1}-\frac{1}{2} \frac{q_{1}^{3}}{q_{2}}\right) p_{2}^{2}+\frac{1}{q_{2}}-\frac{q_{1}^{2}}{q_{2}^{2}} .
\end{aligned}
$$

Then, the Lax representation for $g(\lambda)=1$ takes the form

$$
L=\left(\begin{array}{cc}
-p_{2} \lambda-p_{1}-q_{1} p_{2} & \lambda^{2}+q_{1} \lambda+q_{2} \\
-\frac{\left(p_{1}+q_{1} p_{2}\right)^{2}}{q_{2}}-\frac{2 q_{1}}{q_{2}^{2}}+\frac{2}{q_{2}} \lambda^{-1} & p_{2} \lambda+p_{1}+q_{1} p_{2}
\end{array}\right),
$$




$$
\begin{aligned}
& U_{1}=\left(\begin{array}{cc}
-\frac{1}{2} \frac{p_{1}+q_{1} p_{2}}{q_{2}} \lambda^{-1} & \frac{1}{2}+\frac{1}{2} q_{1} \lambda^{-1} \\
-\left(\frac{1}{2} \frac{\left(p_{1}+q_{1} p_{2}\right)^{2}}{q_{2}^{2}}+\frac{2 q_{1}}{q_{2}^{3}}\right) \lambda^{-1}+\frac{1}{q_{2}^{2}} \lambda^{-2} & \frac{1}{2} \frac{p_{1}+q_{1} p_{2}}{q_{2}} \lambda^{-1}
\end{array}\right), \\
& U_{2}=\left(\begin{array}{cc}
-\frac{1}{2} \frac{q_{1}\left(p_{1}+q_{1} p_{2}\right)}{q_{2}} \lambda^{-1} & \frac{1}{2} \lambda^{-1} \\
-\left(\frac{1}{2} \frac{\left(p_{1}+q_{1} p_{2}\right)^{2}-2}{q_{2}^{2}}+\frac{2 q_{1}^{2}}{q_{2}^{3}}\right) \lambda^{-1}+\frac{q_{1}}{q_{2}^{2}} \lambda^{-2} & \frac{1}{2} \frac{q_{1}\left(p_{1}+q_{1} p_{2}\right)}{q_{2}} \lambda^{-1}
\end{array}\right),
\end{aligned}
$$

for $g(\lambda)=\lambda^{-1}$ we have

$$
\begin{aligned}
& L=\left(\begin{array}{cc}
\frac{p_{1}+q_{1} p_{2}}{q_{2}} \lambda+\frac{q_{1}\left(p_{1}+q_{1} p_{2}\right)}{q_{2}}-p_{2} & \lambda^{2}+q_{1} \lambda+q_{2} \\
-\frac{\left(p_{1}+q_{1} p_{2}\right)^{2}}{q_{2}^{2}}-\left(\frac{q_{1}\left(p_{1}+q_{1} p_{2}\right)^{2}}{q_{2}^{2}}-\frac{2\left(p_{1} p_{2}+q_{1} p_{2}^{2}\right)}{q_{2}}\right) \lambda^{-1} & -\frac{p_{1}+q_{1} p_{2}}{q_{2}} \lambda-\frac{q_{1}\left(p_{1}+q_{1} p_{2}\right)}{q_{2}}+p_{2} \\
-\frac{2 q_{1}}{q_{2}^{2}} \lambda^{-2}+\frac{2}{q_{2}} \lambda^{-3} &
\end{array}\right), \\
& U_{1}=\left(\begin{array}{cc}
0 & \frac{1}{2} \\
\left(\frac{p_{1} p_{2}+q_{1} p_{2}^{2}}{q_{2}^{2}}-\frac{1}{2} \frac{q_{1}\left(p_{1}+q_{1} p_{2}\right)^{2}+2}{q_{2}^{3}}+\frac{2 q_{1}^{2}}{q_{2}^{4}}\right) \lambda^{-1}-\frac{2 q_{1}}{q_{2}^{3}} \lambda^{-2}+\frac{1}{q_{2}^{2}} \lambda^{-3} & 0
\end{array}\right), \\
& U_{2}=\left(\begin{array}{cc}
\frac{1}{2} \frac{p_{1}+q_{1} p_{2}}{q_{2}} & \frac{1}{2} \lambda+\frac{1}{2} q_{1} \\
\left(\frac{q_{1} p_{2}\left(p_{1}+q_{1} p_{2}\right)}{q_{2}^{2}}-\frac{1}{2} \frac{q_{1}^{2}\left(p_{1}+q_{1} p_{2}\right)^{2}+6 q_{1}}{q_{2}^{3}}+\frac{2 q_{1}^{3}}{q_{2}^{4}}\right) \lambda^{-1} & -\frac{1}{2} \frac{p_{1}+q_{1} p_{2}}{q_{2}} \\
+\left(\frac{1}{q_{2}^{2}}-\frac{2 q_{1}^{2}}{q_{2}^{3}}\right) \lambda^{-2}+\frac{q_{1}}{q_{2}^{2}} \lambda^{-3} &
\end{array}\right),
\end{aligned}
$$

and for $g(\lambda)=\lambda$

$$
\begin{aligned}
L & =\left(\begin{array}{cc}
-p_{1} \lambda+q_{2} p_{2} & \lambda^{2}+q_{1} \lambda+q_{2} \\
-\left(\frac{\left(p_{1}+q_{1} p_{2}\right)^{2}}{q_{2}}-p_{2}^{2}+\frac{2 q_{1}}{q_{2}^{2}}\right) \lambda^{2}+\left(2 p_{1} p_{2}+q_{1} p_{2}^{2}+\frac{2}{q_{2}}\right) \lambda-q_{2} p_{2}^{2} & p_{1} \lambda-q_{2} p_{2}
\end{array}\right), \\
U_{1} & =\left(\begin{array}{cc}
\frac{1}{2} p_{2} \lambda^{-2}-\frac{1}{2} \frac{p_{1}+q_{1} p_{2}}{q_{2}} \lambda^{-1} & \frac{1}{2} q_{1} \lambda^{-2}+\frac{1}{2} \lambda^{-1} \\
\left(\frac{p_{1} p_{2}+q_{1} p_{2}^{2}}{q_{2}}+\frac{1}{q_{2}^{2}}\right) \lambda^{-1}-\frac{1}{2} p_{2}^{2} \lambda^{-2} & -\frac{1}{2} p_{2} \lambda^{-2}+\frac{1}{2} \frac{p_{1}+q_{1} p_{2}}{q_{2}} \lambda^{-1}
\end{array}\right), \\
U_{2} & =\left(\begin{array}{cc}
\frac{1}{2} q_{2} p_{2} \lambda^{-2}-\frac{1}{2} \frac{q_{1} p_{1}-q_{2} p_{2}+q_{1}^{2} p_{2}}{q_{2}} \lambda^{-1} & \frac{1}{2} \lambda^{-2} \\
\left(\frac{1}{2} \frac{p_{2}\left(2 q_{1} p_{1}+2 q_{1}^{2} p_{2}-q_{2} p_{2}\right)}{q_{2}}+\frac{q_{1}}{q_{2}^{2}}\right) \lambda^{-1}-\frac{1}{2} q_{1} p_{2}^{2} \lambda^{-2} & -\frac{1}{2} q_{2} p_{2} \lambda^{-2}+\frac{1}{2} \frac{q_{1} p_{1}-q_{2} p_{2}+q_{1}^{2} p_{2}}{q_{2}} \lambda^{-1}
\end{array}\right) .
\end{aligned}
$$

\section{Final comments}

The main result of the article is a systematic construction of a family of non-equivalent Lax representations, parameterized by smooth functions $g(\lambda)$ of spectral parameter, for arbitrary Liouville integrable system defined by separation curve from the class (1.4). We presented in explicit form the Lax matrices $(L, U)$ in separation coordinates $(\lambda, \mu)$ for arbitrary $g(\lambda)$ and in so called Viète coordinates for $g(\lambda)=\lambda^{r}$. It is really astonishing result that any dynamical system (2.2) has so large set of non-equivalent Lax representations. It is still not clear for us what is the geometric meaning of such freedom for the Lax pairs.

In all presented examples, our choice of $g(\lambda)$ was determined only by the simplicity of formulas. In principle there is no obstacles to apply more complex form of $g(\lambda)$, but then all formulas extremely complicate. We did it for the case of two degrees of freedom in Section 3.

It is well known that the knowledge of Lax representation for a given dynamical system allows to construct constants of motion and separation coordinates. So why to study Lax pairs when we start from Liouville integrable and separable system? We were motivated by at least two important reasons. The first one was related with the investigation of the problem of admissible 
structure of Lax pairs. It can be done in a systematic way just in a separation coordinates and led us to the large family of non-equivalent Lax representations. The second one is closely related with our further research program. Actually, it relies on deformation of autonomous Liouville integrable systems, with isospectral Lax representation, to non-autonomous Frobenius integrable systems (Painlevé systems in particular), with respective isomonodromic Lax representation [5].

In order to construct the isomonodromic Lax representation of deformed system the complete knowledge on related isospectral Lax representation is necessary. The work is in progress.

\section{Acknowledgements}

We would like to thank the referees for many useful suggestions and constructive criticisms concerning the first version of this article. Z. Domański has been partially supported by the grant 04/43/DSPB/0094 from the Polish Ministry of Science and Higher Education.

\section{References}

[1] Benenti S., Inertia tensors and Stäckel systems in the Euclidean spaces, Rend. Sem. Mat. Univ. Politec. Torino 50 (1992), 315-341.

[2] Benenti S., Intrinsic characterization of the variable separation in the Hamilton-Jacobi equation, J. Math. Phys. 38 (1997), 6578-6602.

[3] Benenti S., Special symmetric two-tensors, equivalent dynamical systems, cofactor and bi-cofactor systems, Acta Appl. Math. 87 (2005), 33-91.

[4] Błaszak M., Separable systems with quadratic in momenta first integrals, J. Phys. A: Math. Gen. 38 (2005), 1667-1685, arXiv:nlin.SI/0312025.

[5] Błaszak M., Non-autonomous Hénon-Heiles system from Painlevé class, Phys. Lett. A 383 (2019), 21492152, arXiv:1904.05203.

[6] Błaszak M., Sergyeyev A., Natural coordinates for a class of Benenti systems, Phys. Lett. A 365 (2007), 28-33, arXiv:nlin.SI/0604022.

[7] Błaszak M., Sergyeyev A., Generalized Stäckel systems, Phys. Lett. A 375 (2011), 2617-2623.

[8] Eilbeck J.C., Enol'skii V.Z., Kuznetsov V.B., Tsiganov A.V., Linear $r$-matrix algebra for classical separable systems, J. Phys. A: Math. Gen. 27 (1994), 567-578, arXiv:hep-th/9306155.

[9] Marciniak K., Błaszak M., Flat coordinates of flat Stäckel systems, Appl. Math. Comput. 268 (2015), 706716, arXiv:1406.2117.

[10] Mumford D., Tata lectures on theta. II, Progress in Mathematics, Vol. 43, Birkhäuser Boston, Inc., Boston, MA, 1984.

[11] Rauch-Wojciechowski S., Marciniak K., Błaszak M., Two Newton decompositions of stationary flows of KdV and Harry Dym hierarchies, Phys. A 233 (1996), 307-330.

[12] Ravoson V., Gavrilov L., Caboz R., Separability and Lax pairs for Hénon-Heiles system, J. Math. Phys. 34 (1993), 2385-2393.

[13] Tsiganov A.V., Duality between integrable Stäckel systems, J. Phys. A: Math. Gen. 32 (1999), 7965-7982, arXiv:solv-int/9812001.

[14] Vanhaecke P., Integrable systems in the realm of algebraic geometry, 2nd ed., Lecture Notes in Math., Vol. 1638, Springer-Verlag, Berlin, 2001. 